\title{
Biofibre viscosifiers for subsurface production
}

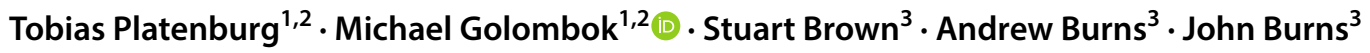

Received: 10 November 2017 / Accepted: 10 May 2018 / Published online: 1 June 2018

(c) The Author(s) 2018

\begin{abstract}
A novel natural cellulose material ("biofibre") suspension in water has potential both for enhanced oil recovery and proppant placement during fracturing in tight reservoirs. We analyse the different carrying mechanisms of biofibre against those of a cross-linked guar benchmark. Three viscosity determination methods have been applied that are relevant to the different applications. Non-Newtonian, shear-thinning effects with viscoelastic enhancement have been observed. These results are compared to the viscosity in a falling ball rheometer as well as in pressure-driven flow through capillaries and conduits. The results indicate that viscosity can be tuned to automatically adjust to the desired flow conditions in the reservoir to provide optimal behaviour for each application.
\end{abstract}

Keywords Fractures $\cdot$ Reservoirs $\cdot$ Fibre suspensions

\begin{tabular}{ll}
\multicolumn{2}{l}{ List of symbols } \\
$A$ & Surface area \\
$\mathrm{d}$ & Diameter ball \\
$D$ & Diameter capillary \\
$K$ & Permeability \\
$L$ & Length \\
$N$ & Crowding number \\
$n$ & Flow behaviour index \\
$P$ & Pressure \\
$Q$ & Flow rate \\
$\mathrm{RF}$ & Resistance factor \\
$u$ & Velocity \\
$v_{\mathrm{t}}$ & Terminal velocity \\
$\mathrm{Greek}$ & \\
$\alpha$ & Comparative parameter \\
$\dot{\gamma}$ & Shear rate \\
$\bar{\gamma}$ & Average shear rate \\
$\Lambda$ & Mass fraction
\end{tabular}

Paper for submission to J. Petrol. Explor. Prod. Tech.

Michael Golombok

michael.golombok@shell.com

1 TU Eindhoven, Groene Loper 15, 5600 MB Eindhoven, The Netherlands

2 Shell Global Solutions Intl. B.V., Grasweg 31, 1031 HW Amsterdam, The Netherlands

3 Pipeline Cleaning Solutions Ltd., Unit J, Isla Bank Mills, Station Rd., Keith AB55 5DD, UK

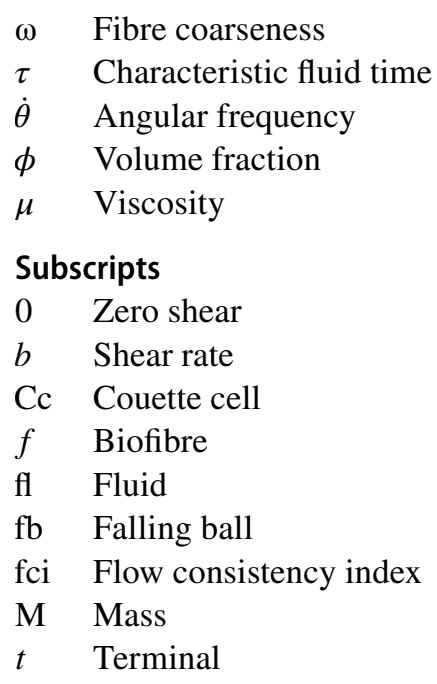

\section{Introduction}

Novel rheology solutions have historically been used in the upstream oil and gas industry for a variety of recovery applications. For "tight" gas reservoirs, a pre-treatment of the reservoir to increase productivity involves the use of viscosity modified fluids. This fracturing process ("fracking") enables reservoir gas production flow (Barati and Liang 2014). The first fracking stage consists of the creation of a wide fracture requiring a low viscosity in the pressurized fluid, followed by a second phase, in which proppant is transported deep into the fracture, which requires a high viscosity. In the third 
stage ("the flowback stage") gas is produced by a reduction in pressure that is followed by the fracture closing on the proppant. This last stage requires a reduced, low viscosity to avoid re-entraining proppant particles during flow back. The entire process requires initially high viscosity fluids with associated viscosifying and subsequently chemical "breakers" to be added to the reservoir. It is the use of these chemicals which have made the process controversial and (so far) unacceptable in Europe.

In oil recovery in fractured reservoirs, shear-thinning fluids are typically used as these are easy to inject (low viscosity at high shear) but display greater viscosity under laminar flow conditions - this aids in reducing the viscosity contrast between the displacing fluid (water) and the oil located in the permeable reservoir. When water is applied as a displacement fluid, a preferential flow path is formed from the injection to the production well, because of spatial variation in permeability over the reservoir. Conformance control aims to promote flow through low permeability zones by preventing flow through high permeability zones using viscositymodified fluids.

There is continuous development of fluid rheologies to improve the desired flow characteristics with better viscosity manipulation as well as to reduce the use of undesirable chemicals. Both goals can be achieved if the rheology is "tuned" to the reservoir and in particular to the distribution of permeabilities or fracture sizes for the oil and tight gas cases, respectively. For the oil recovery problem, the classic enhanced oil recovery current "standard" are gelling polymers which are added to water (Raffa et al. 2016). The gelling effect is time delayed until the solution has penetrated the high permeability portions at which point the solution viscosifies and (hopefully) selectively blocks these volumes. The solutions themselves are shear thinning (for injection purposes) and show a component of rheopectic behaviour (i.e., thickening with time). This is the same characteristic as the guar gum industry standard which is used for the shale gas fracking application.

There are a number of obvious drawbacks to these technologies. For example, the blocking of high permeability zones in the oil recovery scenario is somewhat uneven and could always be better. For the shale gas application, we have already alluded to the undesirable chemical viscosifiers and breakers added to modify viscosity. Recent work on viscoelastic surfactants (Dogon and Golombok 2016a, b), has demonstrated that viscosity modifications can be tuned to arise from the flow conditions associated with the different stages of the process i.e., they display flow-induced viscoelasticity whereby the viscosity adjusts according to the flow conditions. For oil recovery this means that they automatically show high fluid viscous resistance in high permeability regions (Santvoort and Golombok 2018). For the fracking problem it means that they have initially high viscosity for proppant carrying purposes but afterwards during "flowback", the viscosity falls to near water levels enabling return flow through the proppant pack without dislodging particles. This corresponds to a low viscosity at the permeability associated with the proppant pack. (Note that the permeability of such a proppant pack is much higher than the permeabilities associated with the oil production problem discussed above.)

Viscoelastic surfactants are somewhat sensitive to the presence of oil and rather challenging to tune for an unknown fracture size. Moreover, environmental objections to application (such as in the case of shale gas) can best be overcome if the viscosifiers are "natural" products derived without too much processing from plant materials, etc. (Thakur and Thakur 2015) In this paper, we describe a natural material which displays these desired properties. It is a natural cellulose biofibre product which has previously been used for turbulent drag enhancement properties. We demonstrate a natural viscoelasticity which enhances the behaviour over and above current additives used for overcoming permeability variation and proppant placement problems. The material is non-Newtonian-but not just in the timeand shear-dependent manners discussed above-it also has a significant viscoelastic component. This requires careful interpretation of the three-different classic viscosity determination methods with consideration of their applicability to the processes associated with the oil problem (laminar pressure-driven flow) and fracture gas reservoir (combined laminar pressure driven flow and entrained particle flow). In "Background" we discuss previous work on the effect of fibrous solutions on viscosity as well as specifying the viscosity requirements for the oil and gas problem. "Methods" describes the experimental measurements. "Results and discussion" contains our results and discussion and is followed by a conclusion summarizing the main results.

\section{Background}

The earliest description of the effect of suspensions on the viscosity of a base fluid was due to Einstein $(1906,1911)$ who defined an equation for the zero-shear viscosity $\left(\mu_{0}\right)$ for a suspension of hard spheres.

$\mu_{0}=\mu_{b}(1+2.5 \phi)$,

where $\mu_{b}$ is the base fluid viscosity and $\phi$ the volume fraction within the domain $(0<\phi \leq 0.03))$. The equation gains an extra term for spheres that experience hydrodynamic interactions (e.g., drag by a sphere that influences another),

$\mu_{0}=\mu_{b}\left(1+2.5 \phi+6.2 \phi^{2}\right)$.

Due to assumptions made for this equation, it is only valid for low volume fractions $(\phi \leq 0.10)$. It also assumes identical densities for fluid and suspended solid. This relationship 
has been used successfully to model relatively dilute colloidal suspensions such as asphaltene aggregates in heavy oil (Mozaffari et al. 2015). This model needs to be corrected in more sophisticated systems allowing for differing densities of continuous and dispersed phases. Mostly, the mass fraction of solid is used: this is given by:

$\lambda=\frac{\rho_{s} \phi}{\rho_{s} \phi+\rho_{l}(1-\phi)}$.

The specific gravity of cellulose is around 1.5 so the converse of this relationship yields $\phi=\frac{2}{(3 / \lambda-1)}$.

In analogy to Eq. 1 Haward et al. (2012) noted that increasing the fibre mass fraction increases the viscosity due to formation of a complex network and flocs (Karppinen et al. 2012). Fibre suspensions show shear-thinning behaviour-the viscosity decreases due to alignment of fibres under shear. A low power law index $(<0.2)$ is typically found for cellulose fibre suspension (Nazari et al. 2016). Nikbakht et al. (2014) reported a plug flow profile under pressure-driven flow for low velocities, that agrees with low power law indices. These relationships have been classified by Larson (1999) for non-Brownian suspensions where fibres influence each other and cannot rotate freely.

Viscosity increases when the shear mixing time, and thus the amount of energy used, is increased (Shafiei-Sabet et al. 2016). The shear mixing time influences the length and width of the fibres. In oscillatory shear measurements, the viscous modulus was found to be nearly independent of the frequency, while the storage modulus increased with frequency (Derakhshandeh et al. 2010). Viscoelasticity was confirmed with more solid-like behavior for higher fibre concentrations (Zhou et al. 2016). In addition to fibre interactions with water, there are also interfibrous forces dependent on crowding. A crowding number is defined by Kerekes and Schell (1992) as the number of fibres in a suspension swept out by the length of a single fibre.

$N \approx 500 \lambda \frac{L^{2}}{\omega}$,

where $\lambda$ is the fibre mass fraction, $L$ is the weighted average length of the fibres (m) and $\omega$ is the fibre coarseness (weight per unit length of fibre, $\mathrm{kg} / \mathrm{m}$ ).

In shear flow, fibres gather in local mass concentrations known as flocs (Larson 1999). These act as cross-linkers forming a gel. Flocs can have differing mechanical strength. and can cause the suspension to act as a thixotropic fluid. Duffy et al. (2004) noted that flocculation during flow depends on the diameter of the pipe. In smaller diameter pipes, radial floc build-up is restricted. This effect can influence the viscosity for a changing diameter and thus permeability. Studies have proven (Kumar et al. 2016) the existence of a water-rich boundary layers in either shear or (laminar) pressure-driven flow, promoting a lower effective viscosity. Fibres migrate away from the wall resulting in a lower local viscosity. This creates a watery layer at the pipe boundary. (The existence of such a layer implies that Couette cell measurements might not give reliable results, as the assumption is that shear is steadily applied to the entire flow.)

Bulova et al. (2006) focused on polymer-based fibres in a suspension of gels already applied for fracking purposes, such as cross-linked guar gum. Zhao et al. (2016) observed shear-thinning behavior in a rheometer. However, no significant influence on the apparent viscosity by adding fibres to a borate cross-linked fracturing fluid was observed. This was despite a network structure in the gels which might improve the proppant carrying capacity. Yang et al. (2013) were successful in increasing the size of an existing fracture with the addition of fibres. Kusanagi et al. (2015) used cellulose fibres in water up to $0.6 \mathrm{wt} \%$-with cross-linkers-for enhanced oil recovery to yield extra oil from cores, however, injectivity was poor. Sau et al. (2015) suggested the possibility of blocking fractures with fibrous materials for enhanced recovery.

\section{Methods}

\section{Materials}

Cellulose biofibre material ("Uptake", patent: WO2010070354 A1) was obtained from Pipeline Cleaning Solutions Ltd. A suspension is prepared by adding the biofibre to water. Preliminary investigation shows blocking for $\lambda>2 \mathrm{wt} \%$ - mass fractions below this threshold were chosen. Therefore, the mass fractions of biofibre in water were chosen at $0.5,1.0$ and $1.5 \mathrm{wt} \%$. The mixture was prepared using a Silverson L5M-A laboratory mixer equipped with an "emulsion screen". A separate settling study revealed that the best results were achieved for a shear mixing time of $60 \mathrm{~min}$, at a rotational speed of $8000 \mathrm{RPM}$.

As a reference, a borated cross-linked guar gum solution, was prepared that is similar to the standard used by industry for shale gas recovery. Dry guar was acquired from SigmaAldrich together with a borate $0.5 \mathrm{M}$ buffer solution, $\mathrm{pH}$ 9.0. A $0.36 \mathrm{wt} \%$ mass fraction linear guar gum mixture was mixed for $30 \mathrm{~min}$ in a laboratory mixer. Although not essential for the current study, the $\mathrm{pH}$ was increased to 11 after initial mixing, for compatibility with carbonate reservoirs. Borate solution was added to obtain a $95 \mathrm{ppm}$ ion content. At this point, the cross-linking, gelled effect was observed.

\section{Standard viscosity tests}

A Couette cell does not replicate a specific application but is a widely accepted industry standard. The flow and

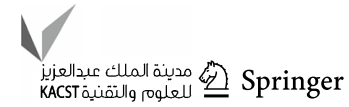


oscillatory viscosity measurements were performed using a TA Instruments Discovery HR-3 Couette rheometer equipped with a standard Peltier concentric cylinder geometry. The cup temperature was maintained at $20{ }^{\circ} \mathrm{C}$. The fluid was pre-sheared for $30 \mathrm{~s}$ at a shear rate of $0.1 \mathrm{~s}^{-1}$. The viscosity measurements for a falling ball used a Thermo Scientific HAAKE Falling Ball (diameter $15 \mathrm{~mm}$ ) Viscometer type C. For each mass fraction, five samples were taken and each sample was measured five times. The resulting viscosity was then averaged. In both cases, we also calibration tested with certified viscosity reference standards as well as known Newtonian standards. The principal source of error in the measurement arises from and is identical to the relative error in the measurement of the time of travel between the two calibration points. This was about $5 \%$ of the measured time and corresponded to the relative errors derived from repeated measurements.

\section{Darcy flow set-up}

The Couette and falling ball viscometers are standard pieces of equipment. The derivation of Darcy viscosity under pressure-driven flow was carried out in a flow cell (Fig. 1). The injection fluid is placed in the intake container (1) and pumped by a Masterflex peristaltic pump (2), which is manually set and calibrated before each measurement. Flow rates are always maintained such that flow is laminar. The pressure drop is measured by a Rosemount 3051CD2-620-620 mbar (accuracy: 0.1\%) differential pressure transducer (4) with connections to the upstream and downstream side of the sample, which was either a capillary or conduit (3).

The capillaries, of diameter $D$, consist of glass tubes with connection pieces to easily attach them to the set-up.

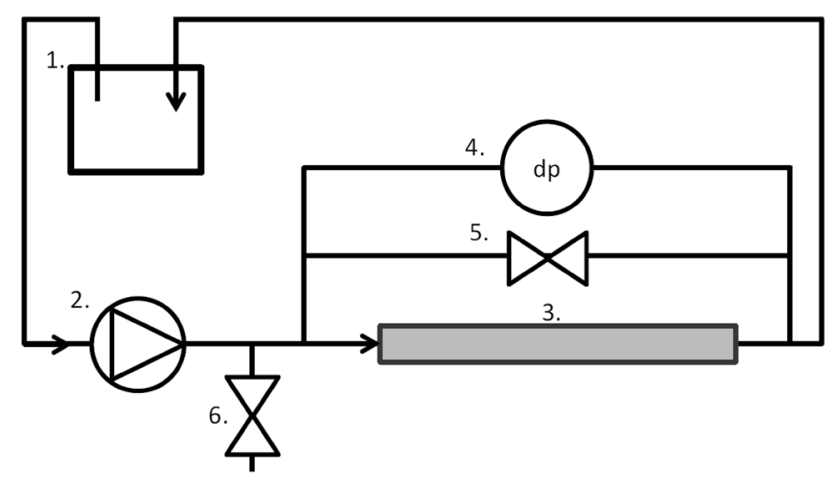

Fig. 1 Schematic overview of the experimental set-up to measure the effective viscosity in pressure-driven flow. The set-up consists of an inlet tank (1), peristaltic pump (2), capillary or conduit (3), differential pressure transducers (4), bypass valve (5) and $\mathrm{CO}_{2}$ flushing valve (6)
The conduit consists of three metal plates, one top and bottom plate with a middle spacer plate that defines the height, $H$, and thus the permeability. The theoretical permeabilities for capillary and conduit respectively are given by (Turcotte and Schubert 1982)

$K_{\text {cap }}=\frac{D^{2}}{32} \quad K_{\text {con }}=\frac{H^{2}}{12}$

Using the measured pressure drop, $\Delta P$, flow rate, $Q$, and permeability, the effective viscosity $\mu$ can be calculated using Darcy's law, defined as:

$\mu=\frac{K A}{Q} \frac{\Delta P}{L}$

with $A$, the surface area, and $L$, the length of the capillary or conduit over which the pressure drop is measured.

The measurement procedure starts with a water flush. The system is de-gassed by opening the bypass valve (5) and valves at the pressure transducer. After this step, the valves are closed and the set-up pump is stopped. Any water is removed from the intake container and the test fluid is flushed through the system a number of times. A flow rate in the range of 50 to $900 \mathrm{ml} / \mathrm{min}$ is set and the pressure drop is allowed to stabilize (ca. $5 \mathrm{~min}$ ). After stabilizing, the differential pressure drop is measured for $5 \mathrm{~min}$ at $5 \mathrm{~s}$ intervals as a function of set flow.

\section{Results and discussion}

\section{Standard viscosity tests}

The viscosity of the biofibre was measured in a Couette cell at three different mass fractions in water: $0.5,1.0$ and

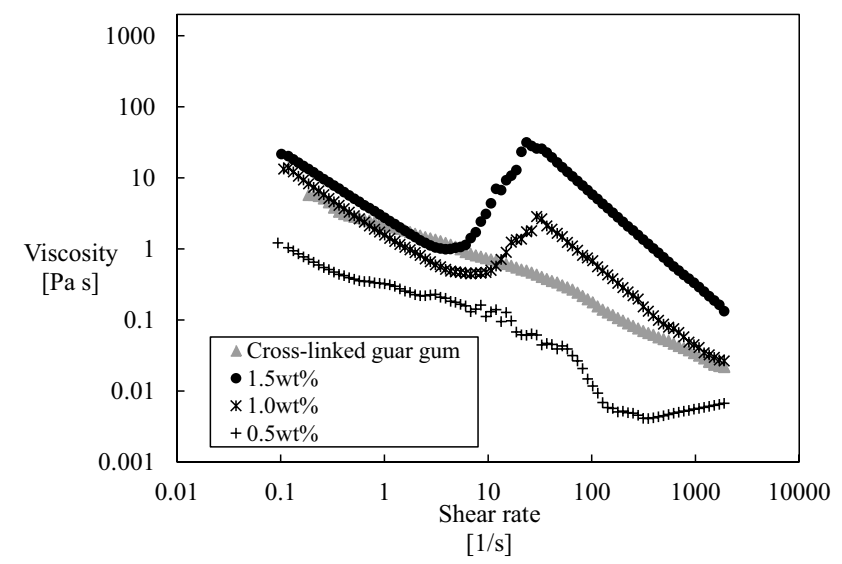

Fig. 2 Characterization of the biofibre at different mass fractions in water and a cross-linked guar gum reference measurement using a Couette cell 
$1.5 \mathrm{wt} \%$ along with the cross-linked guar gum reference (Fig. 2). The viscosity increases with mass fraction of biofibre. All three measured mass fractions show a shear-thinning trend which agrees with previous observations (see 2.1). However, for a mass fraction of $1.0 \mathrm{wt} \%$ and $1.5 \mathrm{wt} \%$, a shear-thickening regime initiates at a shear rate of $10 \mathrm{~s}^{-1}$. Dogon (2016) has shown that this effect is associated with viscoelastic effects. The relationship in Eqs. (1) and (2) breaks down at this point. This is a well-known effect in viscoelastic surfactants forming worm-like micelles (van Santvoort and Golombok 2018). However, as far as we are aware, this is the first time that it has been reported for micron-sized fibres. The application of shear (either by Couette or Darcy flow) begins to align the fibres, however, after a certain threshold they begin to intertangle and this has the effect of increasing the resistance to flow (=viscosity). This effect reaches a maximum at which point mechanical stress breaks down the extended fibre network and the viscosity decreases again.

This transient shear-thickening regime applies over the range 10-30 s $\mathrm{s}^{-1}$ after which shear thinning resumes from the newly enhanced viscosity. The net effect is thus one of shear thinning of viscosity by orders of ca. 100 over a range $0.1-1000 \mathrm{~s}^{-1}$, but with a "hump" interruption of temporary shear thickening between 10 and $100 \mathrm{~s}^{-1}$. This corresponds to the shear rates associated with fracture flow in reservoirs (van der Plas and Golombok 2016). We have previously shown that selective retardation effects in fractures are associated with this transient regime. This has been shown to be chemically tuneable in the case of viscoelastic surfactants-it is as yet not clear how this might be done for these biofibres. For now, we simply note the parallel with viscoelastic surfactants i.e., the occurrence of a transient regime of viscosity thickening which has previously been associated with viscoelasticity.

By contrast, the cross-linked guar gum sample is shear thinning over the entire domain and at low shear is nearly identical to the fibre mass fractions of 1 and 1.5\%. It does not show the shear-thickening regime that initiates at a shear rate of $10 \mathrm{~s}^{-1}$. The measured cross-linked guar gum continues to be shear thinning until the highest shear rate measured at $2000 \mathrm{~s}^{-1}$. In the higher shear rate regime, it shares some characteristics with the $1.0 \mathrm{wt} \%$ biofibre mass fraction. The biofibre solutions, therefore, display some potential viscoelastic advantage over cross-linked guar gum. As shown by the $\log -\log$ plots in Fig. 1a, the behaviour is of the form

$\mu=\mu_{0} \dot{\gamma}^{n-1}$

This relationship is of limited utility given the onset of the shear-thickening regime interruption due to viscoelasticity. We confine ourselves to the observation that the index $n=0.2$ is lower for biofibre than for guar gum $(n=0.4)$. Biofibre is thus more shear thinning and has more potential for improved reservoir injectivity compared to cross-linked guar gum. This obviates the need for time-delayed crosslinkers because we can rely on the natural reduced viscosity at the high shear associated with injection to provide sufficient flow.

Moving towards proppant transport, the viscosities were also measured in a falling ball viscometer (Fig. 3). Entanglement of fibres is more at higher concentrations, increasing the resistance for the ball to fall through. The viscosity increases by a factor 10 over the biofibre mass fraction range-much higher than suggested by Eqs. (1) and (2). (The falling ball viscosity for the cross-linked guar gum reference was almost two orders higher than for biofibre $-720 \mathrm{mPa} \mathrm{s}$ ). Note that the falling ball has a diameter of $15 \mathrm{~mm}$ - an order of magnitude higher than the size of typical proppant particles. The falling ball viscometer is not associated with a specific shear rate, as opposed to the Couette cell. For Newtonian fluids, identical viscosities are of course obtained. For non-Newtonian fluids, the viscosity depends on the experienced shear rate: an average shear rate for the falling ball can be defined by:

$\bar{\gamma}=\frac{2 v_{t}}{d}$

with terminal velocity $v_{t}$ and the diameter of the ball, $d$. This results in an average shear rate range of $7-14 \mathrm{~s}^{-1}$. We use the viscosity at a shear rate of $10 \mathrm{~s}^{-1}$ from the Couette results in Fig. 2 and plot these as a function of concentration in Fig. 3. The Couette viscosities are, like the Guar gum reference, several magnitudes higher than the falling ball. This apparent anomaly is resolved if we note that the terminal velocity (ignoring buoyancy) is inversely proportional to the square root of the diameter i.e., $\mathrm{v}_{\mathrm{t}} \propto \sqrt{ } \mathrm{d}$. This then gives the scaling factor for the shear as $\bar{\gamma} \sim 1 / \sqrt{ } d$. If we replace the apparent shear (Eq. 8) of the $15 \mathrm{~mm}$ ball with that which would be experienced by, for example, a $1.5 \mathrm{~mm}$ (more representative

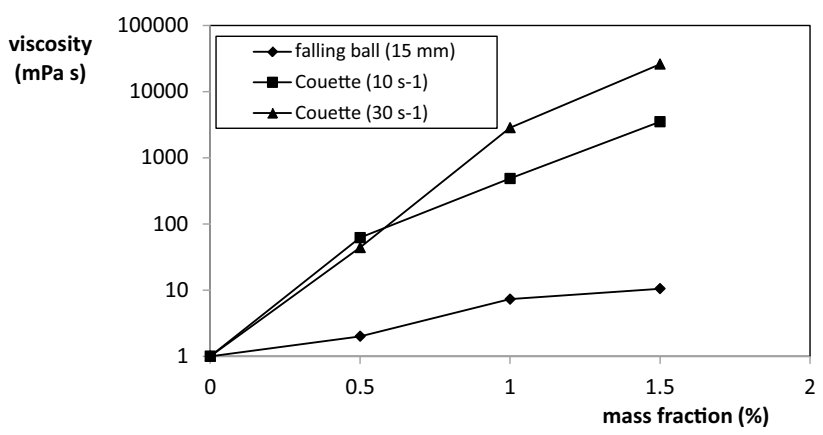

Fig. 3 Falling ball and Couette viscosity (at matched average shear) for various concentrations of biofibre in water. $10 \mathrm{~s}^{-1}$ corresponds to the $15 \mathrm{~mm}$ falling ball. $30 \mathrm{~s}^{-1}$ applies to proppant dimension particles. See text for full discussion

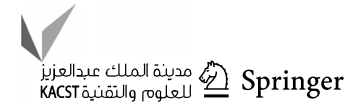


of real proppant dimensions) then we find ourselves at around a shear value of $30 \mathrm{~s}^{-1}$. Turning to Fig. 2 we see that we are in the middle of the viscoelastic "hump" which has been identified above for the biofibre. Figure 3 shows the extrapolated behaviour for such a proppant size particle. The viscosity now deviates upward by an order of magnitude for concentrations above $0.5 \%$. We can expect a similar order of magnitude increase for these particles in falling ball mode. (This is quite apart from any effects due to the ensemble of many proppants.) The reason for this effect is purely the viscoelastic enhancement shown by biofibres and not obtainable with guar gum.

The viscoelastic peak can be specifically accessed by oscillatory rheology. A sinusoidal shear deformation was applied at a frequency increasing from 1 to $100 \mathrm{rad} / \mathrm{s}$ and the resulting stress response was measured. The viscoelastic behaviour can be characterized by a storage modulus $\left(G^{\prime}\right)$ and loss modulus $\left(G^{\prime \prime}\right)$ that indicate the solid-like and fluidlike behaviour, respectively, as described by Weitz et al. (2007). Figure 4a shows the storage modulus and loss modulus for a strain of $0.5 \mathrm{rad}$ and $0.05 \mathrm{rad}$ for biofibre $1.0 \mathrm{wt} \%$. The loss moduli are relatively constant for both strains except at high frequencies (shear rates). (This also applies to the storage modulus for the low strain measurement.) This significant fluctuation is associated with the storage modulus at the higher strain. This indicates that over most of the range the solution is viscous since $G^{\prime \prime}>>G^{\prime}$. However, a cross over occurs at a frequency of $40 \mathrm{~s}^{-1}$ (indicated by the dotted line). This change from fluid-like viscous to solid-like elastic behaviour is typical for viscoelastic solutions where
Fig. 4 a Storage and loss modulus as a function of frequency for biofibre $1.0 \mathrm{wt} \%$ at a strain of $\theta=0.5$ and $\theta=0.05$ and $\mathbf{b}$ the corresponding characteristic fluid times of biofibre at different mass fractions in water

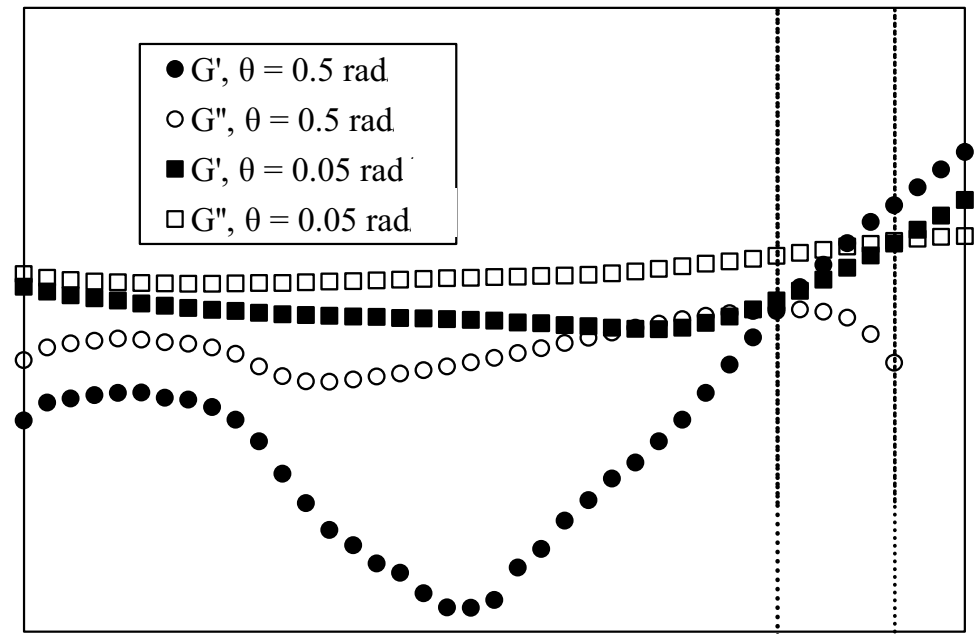

(a)

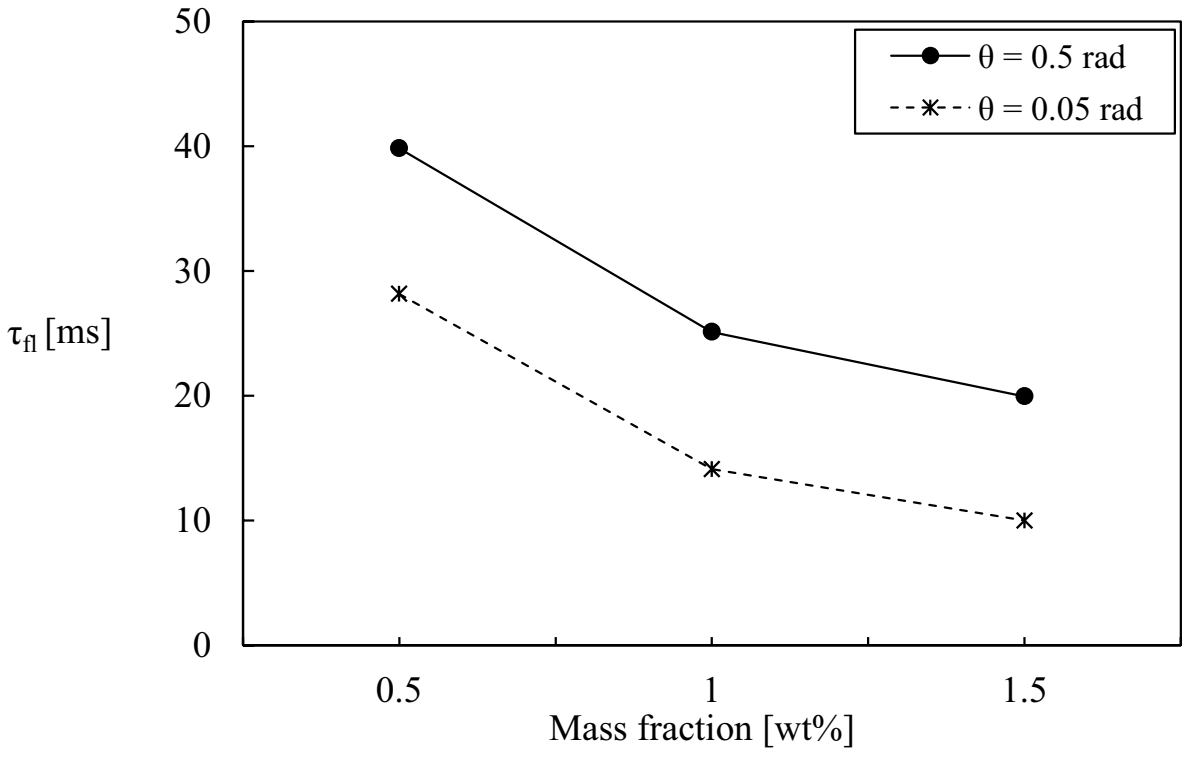

(b) 
it is associated with shear-induced structures. By analogy, we argue that shear leads to fibre entanglement leading to a "springier" response of the fluid than would be expected. Ultimately, as shear increases though, the structures will not be able to sustain this and the fibres align classically with the flow as we saw in Fig. 2 above. The corresponding cross over for the lower strain of 0.05 rad requires a higher rate and this occurs at $71 \mathrm{rad} / \mathrm{s}$ (indicated by the second dotted line). The characteristic fluid time, $\tau_{\mathrm{ff}}$, can be calculated for each cross-over point as,

$\tau_{\mathrm{fl}}=\frac{1}{\dot{\theta}\left(\tan (\delta)=\frac{G^{\prime}}{G^{\prime \prime}}=1\right)}$

with $\dot{\theta}$ the angular frequency at $\tan (\delta)=1$, defined by an equal storage and loss modulus. In accordance with previous studies of the Deborah number of these solutions (Dogon and Golombok 2016b), $\tau_{\mathrm{fl}}$ is lower for lower strains, i.e., at smaller strains the solutions are less "fluid". This suggests that higher strains promote fibre entanglement and formation of complex networks, resulting in a more solid-like behaviour.

\section{Pressure-driven flow}

The effective viscosity was measured in laminar pressuredriven flow using the Darcy flow set-up discussed in "Methods". A velocity vs. pressure drop response is shown in Fig. 5a for a $1.0 \mathrm{wt} \%$ biofibre mass fraction. This shows a non-linear increase of velocity over the pressure drop (seen for all mass fractions). A non-dimensionalized viscosity can be defined by the introduction of a resistance factor, which is demonstrated by Sorbie (1991) (Fig. 6). The resistance factor is defined as

$R F=\left(\frac{\Delta P_{f}}{\Delta P_{0}}\right)_{u}=\left(\frac{\mu_{f}}{\mu_{0}}\right)_{u}$

where $\Delta P_{f}$ is the pressure drop in the experimental set-up with biofibre and $\Delta P_{0}$ the pressure drop of the base fluid of biofibre solution, i.e., water. This results in a dimensionless ratio between the viscosity of biofibre, $\mu_{\mathrm{bf}}$, and water, $\mu_{0} \approx 1 \mathrm{mPa}$ s. at a constant flow velocity so that the number corresponds to the effective viscosity in $\mathrm{mPa}$ s. This nonlinear velocity increase corresponds to a non-linear decrease in viscosity plotted in Fig. 5b.

It was difficult to obtain overlap in pressure, however, overlap does exist for different flow velocities as indicated in Fig. 5 enabling application of the resistance factor given in Eq. 10. As we are concerned with higher flows for both the proppant placement and fracture flow control application, we choose the higher $(6 \mathrm{~cm} / \mathrm{s})$ velocity to demonstrate the effective viscosity as a function of permeability for a conduit and capillary flow (Fig. 6). The former is more realistic for application purposes and indicates a constant viscosity with the guar gum reference having the highest flow viscosity - although this is of course with full crosslinkage prior to viscosity breaking.

The Couette rheometer values of viscosity are much higher than for the falling ball or Darcy flow, e.g., for biofibre $1.5 \mathrm{wt} \%$ values of $0.1-28 \mathrm{~Pa}$ s are measured in the Couette cell whereas in Darcy fw it is $4-40 \mathrm{mPa}$ s. Note that in both Darcy and falling ball methods, there are a wide range of shear rates present whereas the Couette cell subjects the fluid to a single shear rate at any time (Reuvers and Golombok 2008). The wall shear rate is an indicator for a bulk shear rate in Darcy flow and this yields a range of 50-3000 s-1. For the lower mass fractions, higher shear rates were present at the wall and these better match the falling ball and Couette viscosities. For example, for biofibre $0.5 \mathrm{wt} \%$, a viscosity of $3.6 \mathrm{mPa}$ s was measured in a conduit of $2 \mathrm{~mm}$. This corresponds with a wall shear rate of about $400 \mathrm{~s}^{-1}$, which for the Couette rheometer results in a viscosity of about $4 \mathrm{mPa}$. The viscosities are similar for falling ball and pressure-driven flow results. Both share the characteristic application of different shear rates to the material. For the cross-linked guar gum, the viscosity in Darcy flow seems to be lower, which is probably due to a higher average shear rate.

Finally, we note that in this study, we have not specifically considered temperature effects. The study here was conducted at room temperature, however, for subsurface application we need to consider the effect of the elevated temperatures associated with reservoirs in the range $60{ }^{\circ} \mathrm{C}$. Viscoelastic surfactant solutions are notorious for their loss of effect as the temperature is raised. This is because they consist of molecular assemblies such as worm-like micelles which disintegrate as soon as the temperature is raised, i.e., they are Brownian suspensions. In theory, the applied shear effects disturb the equilibrium which is restored at rates defined by the diffusivity (Larson 1999). This is determined by the Stokes-Einstein relationship to be proportional to temperature. However, in our case, we are above the concentration, size and aspect ratio of fibres associated with dilute Brownian behaviour. Nonetheless, there is an analogy here with worm-like micelles in that induced shear leads to some entanglement in the fibres which contributes to the viscoelastic effects we have observed. The behaviour under enhanced temperatures is the subject of current study.

The basic structures are geometrically similar. Worm-like micelles are analogous to microfibres. The molecules making up the micellar chain in the former are easily aligned but also broken up by shear. The fibres, however, consist of fixed permanent saccharides solidly attached by strong covalent

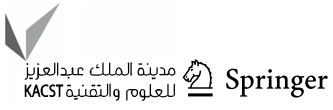


Fig. 5 a Velocity and b effective viscosity as a function of pressure drop for flow through a conduit of three different heights for biofibre at $1.0 \mathrm{wt} \%$ in water

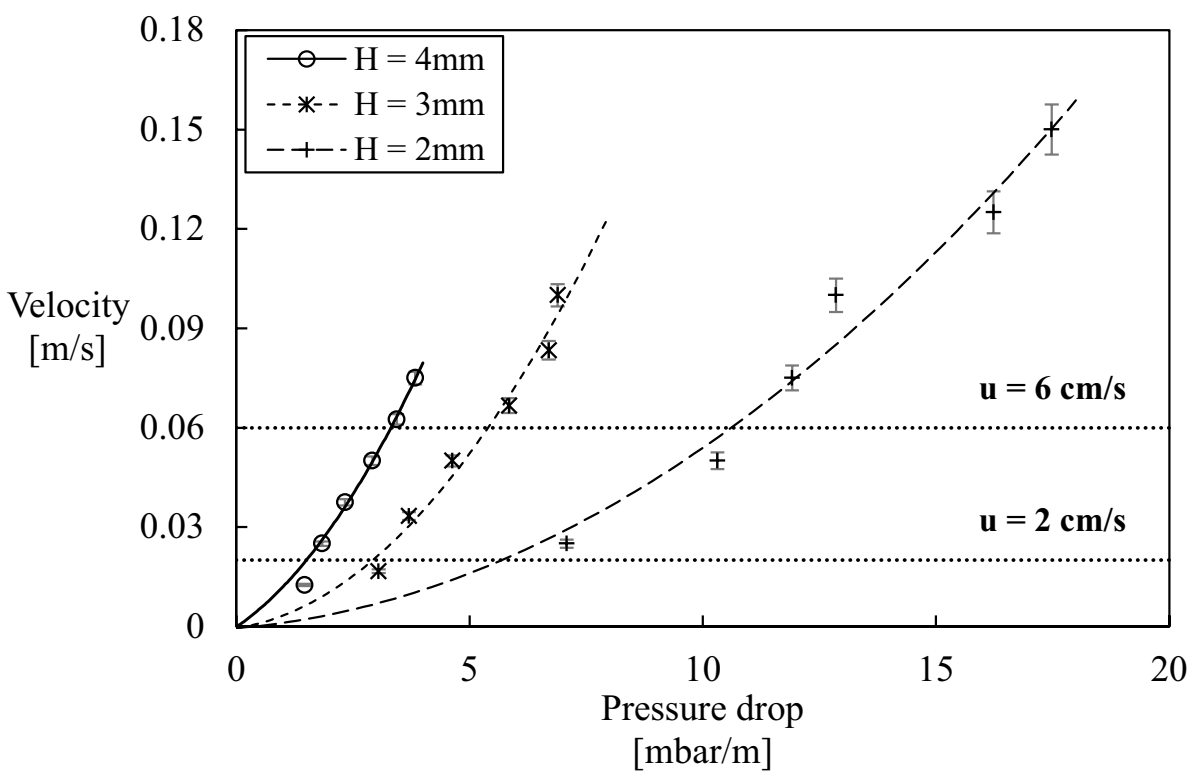

(a)

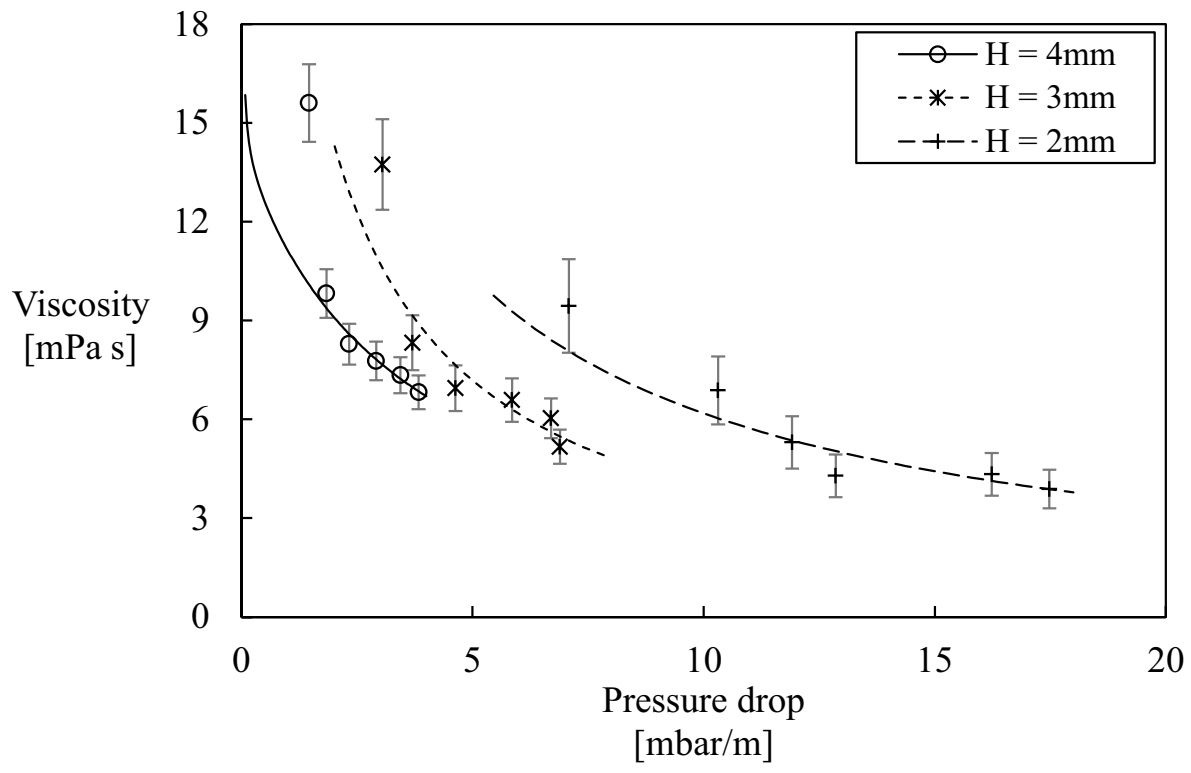

(b) bonds. Unlike worm-like micelles, they are not held together merely by transient polar attraction. The fibres are similar to the worm-like micelles, however, in that they respond to flow-induced forces. This implies that they are less affected by temperature (see below).

\section{Conclusion}

1. Cellulose "biofibre" suspensions show shear-thinning behaviour with a range of intermediate shear thickening associated with viscoelasticity. The different (shear thin- ning and shear thickening) regimes agree with a power law model.

2. The viscosities in the falling ball and Darcy flow tend to be 1 to 2 orders of magnitude lower than the Couette equivalents, even at the high shear ends of the shearthinning curves.

3. Increasing fibre concentration affects Couette viscosity rather more than the application representative viscosity measurement (i.e., falling ball and Darcy)

4. The viscoelastic effect is confirmed with elastic effects predominant at frequencies above $40-70 \mathrm{hz}$ corresponding to the enhancements in steady state Couette flow. 


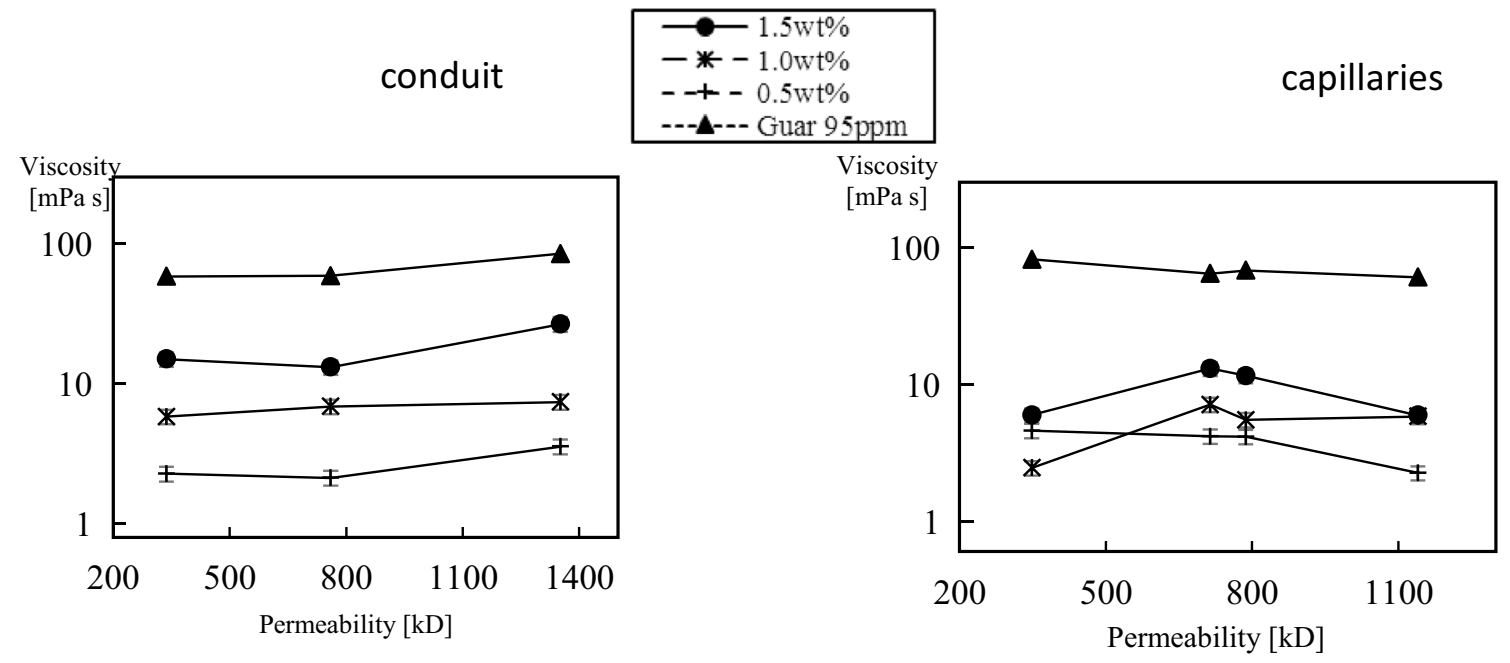

Fig. 6 Effective viscosity in pressure driven flow at a velocity of $6 \mathrm{~cm} / \mathrm{s}$ for $\mathbf{a}$ a conduit and $\mathbf{b}$ capillaries a function of permeability for biofibre at different mass fractions in water and a cross-linked guar gum reference measurement

5. There is clearer differentiation at different concentrations for conduit rather than capillary flow. This suggests a surface volume effect which could be fruitfully explored by further increasing concentration.

6. The viscoelastic effects suggest application in highly fractured reservoirs or fracking in tight reservoirs since the flow response obviates any need for cross-linkers or viscosity breakers. (Control of permeable matrix flow is not possible with this material as it cannot permeate porous matrix - and thus no filter cake is formed as often happens with polymer materials.)

7. Further work is aimed at assessing temperature effects: these materials are much less sensitive to increased temperature which make them easier to apply under reservoir conditions. This is expected to provide considerable advantage when ensembles of particles are used.

Open Access This article is distributed under the terms of the Creative Commons Attribution 4.0 International License (http://creativeco mmons.org/licenses/by/4.0/), which permits unrestricted use, distribution, and reproduction in any medium, provided you give appropriate credit to the original author(s) and the source, provide a link to the Creative Commons license, and indicate if changes were made.

\section{References}

Barati R, Liang J (2014) A review of fracturing fluid systems used for hydraulic fracturing of oil and gas wells. J Appl Polym Sci 131(16):40735

Bulova MN, Nosova KE, Willberg DM, Lassek JT (2006) Benefits of the novel fiber-laden low-viscosity fluid system in fracturing lowpermeability tight gas formations. Society of Petroleum Engineers
Derakhshandeh B, Hatzikiriakos SG, Bennington CPJ (2010) Rheology of pulp suspensions using Doppler velocimetry. Rheol Acta 49(11):1127-1140

Dogon D (2016) Particle flow in fractures, PhD thesis, Eindhoven University of Technology

Dogon D, Golombok M (2016a) Wellbore to fracture proppant-placement-fluid rheology. J Unconventional Oil Gas Resour 14:12-21

Dogon D, Golombok M (2016b) Self-regulating solutions for proppant transport. Chem Eng Sci 148:219-228

Duffy GG, Ramachandra S, Xu JQ (2004) Developments in processing fibre suspensions. In: Proceedings of the fifty-eighth appita annual conference and exhibition, vol. 2, 433-442

Haward SJ, Sharma V, Butts CP, McKinley GH, Rahatekar SS (2012) Shear and extensional rheology of cellulose/ionic liquid solutions. Biomacromolecules 13 (5), 1688-1699

Karppinen A, Saarinen T, Salmela J, Laukkanen A, Nuopponen M, (2012) Flocculation of microfibrillated cellulose in shear flow. Cellulose 19:1807-1819

Kerekes RJ, Schell CJ (1992) Characterization of fibre flocculation by a crowding factor. J Pulp Pap Sci 18(1):32-38

Kumar V, Nazari B, Bousfield D, Toivakka M (2016) Rheology of microfibrillated cellulose suspensions in pressure driven flow. Appl Rheol 26:43534

Kusanagi K, Murata S, Goi Y, Sabi M, Zinno K, Kato Y, Liang Y, (2015) Application of cellulose nanofiber as environment-friendly polymer for oil development. Society of Petroleum Engineers

Larson RG (1999) The structure and rheology of complex fluids (topics in chemical engineering). Oxford University Press, Oxford, ISBN: 9780195121971

Mozaffari S, Tchoukov P, Atias J, Czarnecki J, Nazemifard N (2015) Effect of asphaltene aggregation on rheological properties of diluted Athabasca bitumen. Energy Fuels 29:5595-5599

Naderi A, Lindström T (2016) A comparative study of the rheological properties of three different nanofibrillated cellulose systems. Nordic Pul Pap Res J 31(3), 354-363

Nazari B, Kumar V, Bousfield DW, Toivakka M (2016) Rheology of cellulose nanofibers suspensions: boundary driven flow. J Rheol 60(6):1151-1159

Nikbakht A, Madani A, Olson JA, Martinez DM (2014) Fibre suspensions in Hagen-Poiseuille flow: transition from laminar plug flow to turbulence. J Nonnewton Fluid Mech 212:28-35 
Plas BA van der, Golombok M (2016) Suppressing fluid loss in fractures. J Pet Explor Prod Tech 6(1):85-92

Raffa P, Broekhuis AA, Picchioni F (2016) Polymeric surfactants for enhanced oil recovery: a review. J Petrol Sci Eng 145:723-733

Reuvers N, Golombok M (2009) Shear rate and permeability in water flooding. Transp Porous Media 79(2):249-253

Santvoort J van, Golombok M (2018) Improved recovery from fractured reservoirs. J Petrol Sci Eng 167:28-36

Sau R, Shuchart C, Clancey B, Lecerf B, Pavlova S, (2015) Qualification and optimization of degradable fibers for re-stimulation of carbonate reservoirs. International Petroleum Technology Conference, 2015

Shafiei-Sabet S, Martinez M, Olson J (2016) Shear rheology of micro-fibrillar cellulose aqueous suspensions. J Cellulose 23(5):2943-2953

Sorbie KS (1991) Polymer-improved oil recovery. Springer Science + Business Media New York

Thakur VK, Thakur MK (2015) Handbook of Sustainable Polymers: Processing and Applications. Pan Stanford Publishing, Singapore, 9789814613538

Turcotte DL, Schubert G (1982) Geodynamics-applications of continuum physics to geological problems. Wiley, New York
Weitz D, Wyss H, Larsen R (2007) Oscillatory rheology: measuring the viscoelastic behaviour of soft materials. GIT Lab J Eur 11:68-70

Yang S, Fujian Z, Xianyou Y, Xiongfei L, Shengjiang L, Xiangdong L (2013) Laboratory study and field application of fiber-based fracture reorientation technology. International petroleum technology conference

Zhao Z, Ma J, Guo J, Gao Y, Omeiza AA (2016) Experimental investigation of rheological properties of fiber-laden crosslinked fracturing fluids. J Nat Gas Sci Eng 32:28-34

Zhou L, He H, Li M, Song K, Cheng HN, Wu Q (2016) Morphological influence of cellulose nanoparticles (CNS) from cottonseed hulls on rheological properties of polyvinyl alcohol/CN suspensions. Carbohyd Polym 153:445-454

Publisher's Note Springer Nature remains neutral with regard to jurisdictional claims in published maps and institutional affiliations. 